\title{
Ultrasound Speed of Polymer Gel Mimicked Human Soft Tissue within Three Weeks
}

\author{
Nur Shakila Othman, Muhamad Suhaimi Jaafar, Azhar Abdul Rahman, Ernee Sazlinayati Othman, and \\ Aifa Afirah Rozlan
}

\begin{abstract}
A Polymer gel mimicked human soft tissue was being fabricated using a monomer named 2-Hydroxyl-Ethyl-Acrylate (HEA) with the present of gelatin. The readymade gel which is the concentration for HEA fixed at $5 \%$ was then undergoes an ultrasonic evaluation to test for the propagation of sound speed through it. Sonic Waves Analyzer is absolutely the most accurate technique to determine the aims (changes of speed as function of polymer structure /stiffness over time). The fixed frequency of transducers involved is at 500 $\mathrm{kHz}$ by using the seismic reflection concept. In the observation of relationship between the ultrasound propagation speeds as the day increased, the propagation speed still varies between 1390 to $1500 \mathrm{~m} / \mathrm{s}$ which is still in the range of speed of sound for human tissue.
\end{abstract}

Index Terms-Tissue mimicking, ultrasound phantom, abdominal ultrasound, ultrasound propagation speed

\section{INTRODUCTION}

The ultrasound propagation speed of polymer gels was being characterized. Ideally, the word 'mimics' here can be explained as simulating the specific part of human tissue or this simulation purpose more familiarizes called as phantom. Tissue-mimicking phantom serve an important role in ultrasound research and development without the required to use human or animals in experiment. The fabrication of the gel used monomer type 2-Hydroxyl-Ethyl-Acrylate (HEA), gelatin as the gelling agent to control the phantom stiffness [1] and ascorbic acid functioning as the anti-oxidant. The special design was created and fabrication of this tissue equivalent phantom should mimics human soft tissue. Tissue mimicking phantom also is a substitute of any material that simulates a body tissue.

The phantom used for characterization and calibration of US imaging system. The evaluation of accuracy and performance of ultrasound machine systems usually involve tissue mimicking phantoms.

In the range of medical application, there are some tissues

Manuscript received August 20, 2011; revised September 5, 2011.

Nur Shakila Othman was born in Perak, Malaysia in 1987.The author obtained the Bachelor Degree of Applied Science (Medical Physics) in 2009 from the Universiti Sains Malaysia (USM) Penang. The next year later, she recently was furthering her study in Master degree in Medical Physics programmed specializing in ultrasound.

M. Suhaimi Jaafar received B.Degree in Physics, Mathematics (Aston) in 1980, M.degree in Physics (Aston) in 1982 and Phd Degree in Instrumentation, Analytical Science in 1986 from Manchester.

Azhar Abdul Rahman received his B. Degree in Nuclear Physics from University Kebangsaan Malaysia in 1997. He obtained the M. degree in X-ray Crystallography from University Sains Malaysia in 2004 and Phd Degree in Universiti Putra Malaysia. phantoms available commercially [2]. The comparison between the multipurpose manufactured phantoms such as (Zerdine ${ }^{\mathrm{TM}}$ from CIRS Inc., condensed-milk-based gel from Gammex RMI and urethane-rubber-based from ATSLabs)[3]were made, this fabricated phantom has several advantages, i.e the phantom is easily made, low cost, less fragile, and the preparation using less hazardous chemical, and can last within three weeks ( 23 days) based on visual inspection from the ultrasound image and direct observation.

However, there are lots of tissues mimicking materials for ultrasound phantoms, including agar (polysaccharide), oil gel [4], polyvinyl alcohol gel (PVA) [5], poly acryl amide gel (PAA) [6],

Since 1993, polymeric materials have been used widely in many industries. Among them are transportation, energy production, agriculture, the building industry, retail, packaging consumer goods, foods, and the health and pharmaceutical industries. Some types of polymer like polyolefin, poly (vinyl chloride), polystyrene and synthetic rubbers have been used for more than half of the century in such industrial marketing. Monomer is the core materials in the polymer formation. There are several types of monomers have been used as tissue mimicking material such as acrylamide, acrylic acid, meth acrylic acid, 2-hydroxyl-methyl-acrylate (HEMA), 1-vinyl-2 pyrrolidinone, and including 2-hydroxyl-ethyl-acrylate (HEA).The previous study has also using HEA for biomedical application [4] especially as hydrogel but the important thing is the gel need to polymerize to obtain polymers.

Polymer gel phantom were manufactured consisting of 5\% (by volume/weight) 2-Hydroxylethyl Acrylate (HEA), 3\% N,N' - Methylene - bis - Acrylamide comonomers dissolve in aqueous gelatin (5\% gelatin by total weight and $89 \%$ de-ionized water). By using the ultrasound machine, the imaging evaluation was applied to the gel to ensure whether it can simulate the real condition of human soft tissue similar like the several readymade phantoms (manufactured) depend on the image obtained from the screen.

At constant room temperature, the most important thing is the speed of sound through the gel must be in the range of human tissue $1460 \mathrm{~m} / \mathrm{s}$ to $1650 \mathrm{~m} / \mathrm{s}$ [7] within three weeks (21 days). The measurement for ultrasound speed was made by using Sonic Waves Analyser (SWA). The Multichannel Analyser (MCA) software and collimated radiation beam photon from $\mathrm{a}^{241} \mathrm{Am}$ source were used to measure the linear attenuation coefficient, $\mu$ of the polymer gel [8]. Furthermore, density measurement showed that this polymer gel phantom is equivalent to human soft tissue. This polymer gel tissue 
phantom still undergoes some characterizations and these preliminary results proved that the polymer gel is equivalent to human tissue.

The acoustic properties were measured by using a through-transmission water-substituting method. Regarding to the absolute results of mechanical and acoustic properties; the copolymer-in-oil phantom is equivalent with soft tissue. The results for the ultrasound speed and density reported were from $1420-1464 \mathrm{~m} / \mathrm{s}$ and $0.90 \pm 0.04 \mathrm{~g} / \mathrm{cm}^{3}$ respectively. The fabricated phantom materials used here consist of mineral oil and mixture of copolymer, completed with some additives for acoustic scattering purposed [9].

\section{MATERIAL AND METHOD}

\section{A. Polymer gel dosimeter manufacture}

Preparation of polymer gel phantoms by using 5\% (by volume)(HEA) (Sigma Aldrich, ) completed by comonomers, $3 \%$ N,N' - methylene - bis -acrylamide (BIS) (Sigma) dissolved in aqueous gelatin ( $8 \%$ gelatin by total volume) and $84 \%$ of de-ionized water. After the production finished, the gel were left to cool down to room temperature $\left(22{ }^{\circ} \mathrm{C}\right)$ by maintaining the stirring rate. The gel were poured into ependorf tube, sealed with parafilm tape (Sigma) (to minimize the oxygen contamination inside gel) for linear attenuation coefficient measurements or into small empty vials for density and speed of sound measurement. Then, the remainder gels were poured into the designed container for ultrasound diagnostic imaging. Lastly, those three samples with different shape were then kept in a refrigerator with appropriate condition at approximately $10{ }^{\circ} \mathrm{C}$ for 2 hours until a visual detection concluded that the gel already solidified.

\section{B. Ultrasound Propagation Speed Measurements}

Ultrasound is the name given to high-frequency sound waves, which are exactly greater than human hearing range. The frequency is beyond the audible frequency range which is $20,000 \mathrm{~Hz}$ or higher. As shown in Figure 1, the measurement was done by using the Sonic Waves Analyzer (SWA) under room temperature with the assistance of TDS 1012B Oscilloscope completed by LABVIEW software. The SWA consist of two transducers which act as transmitter and receiver. The sound will be reflected back to the transducer and the remainder will travel through the gel. This is a pulse echo principle from the sequence of events. The propagation speed is the speed measured at whenever the sound moves through a medium which depends on the density of the gel. Generally, the value is highest in solids, average in liquids and lowest in gases.

The longitudinal speed in the pulse echo mode is defined as

$$
v=d / t
$$

where $v=$ longitudinal speed, $\mathrm{m} \mathrm{s}^{-1}, d=$ thickness of gel in vial, $\mathrm{m}, t=$ time of flight [10]

There are two $6 \mathrm{~mm}$ transducers involve in this experimental method, transmitter and receiver. The transducers were aligned properly, and the vial occupied with the gel, was located in between those transducers. Before the measurement started, the coupling transmission gel was covered by all the sensor area of transducer. Then, a dampening circuit was used to reduce the transmitted signal length to a sinusoid of one cycle. The maximum reflected signal received by the transmitting transducer is $500 \mathrm{kHz}$ in frequency. Each signal detected by transducers was displayed on a digitizing oscilloscope (Tektronics TDS 1012B). The sinusoidal signal from oscilloscope was digitized and transferred to a PC for some processing steps such as some noise of the waves were filtered by using special LabView software (version 2.5.1). The repetitions were done three times to determine the ultrasound pulse echo (time of light, $t$ ) from the average signal. The propagation speed was calculated with the time of light, $t$, obtained from the waves generated to PC.

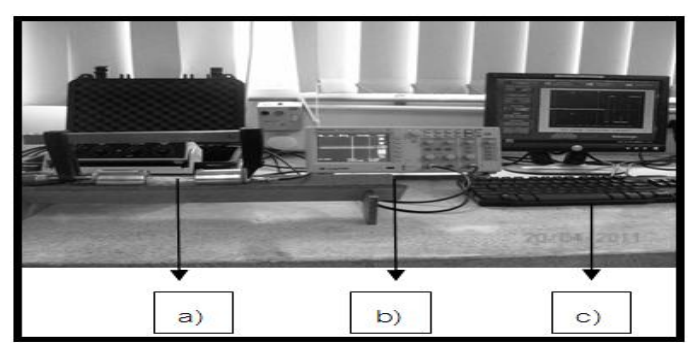

Fig. 1(a). Sonic Wave Analyzer (b) Oscilloscope c) PC with LabView Software

\section{RESULTS AND DISCUSSION}

Based on the results, the propagation speed increase for the first seven days and maintain in the range between 1390 to $1500 \mathrm{~m} / \mathrm{s}$ within 16 days afterwards. This gel is without sound scatter additives. The density of the sample increased as the day increased. This is may be due to some changes in the elastic properties of the polymer gel, which given as $K$, bulk elastic modulus once mentioned by V A et al 1997 in [11]:

$$
K=v^{2} p
$$

where $p$ is bulk density and $v$ is acoustic speed. Elastic properties of the material (gel) are also able to be known here as the compressibility of the material. Regarding to equation (2), an increase in elastic modulus will result in increase in the acoustic speed, compared to density because the compressibility plays more important role to determine the sound speed through media An increase in density which should leads to decrease in the propagation speed as in Figure 2 (a), actually didn't affect much of the value of the sound speed. Every five days each, the physical condition of those polymer gels was being observed while the sound speed was measured. As the day increased, the result proved that there are some changes in polymer structure based, in other words, the polymerization was occurred (formation of cross-linked) naturally and absolutely tends to increase the elastic modulus and also rigidity inside the gel. The value of acoustic speed is not expected to be fluctuated in case there is any distorted of ultrasonic pulse through the gel in the experimental procedure. 


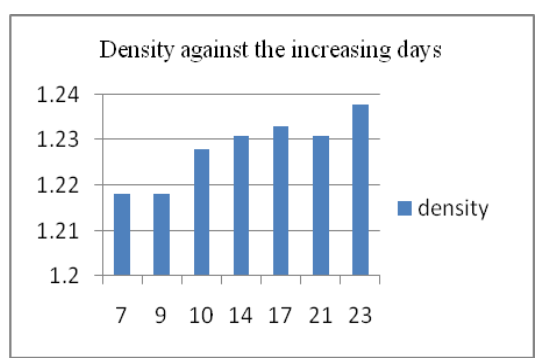

Fig. 2 (a).Graph of density against the increasing days

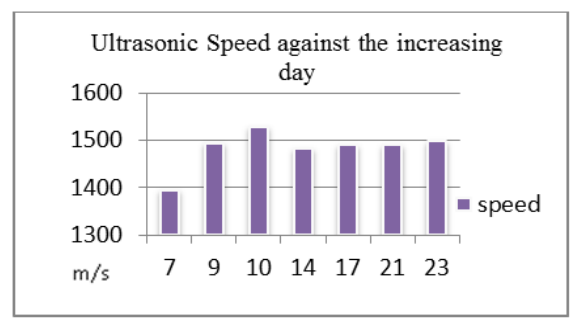

Fig. 2 (b).Graph of ultrasonic speed against the increasing days

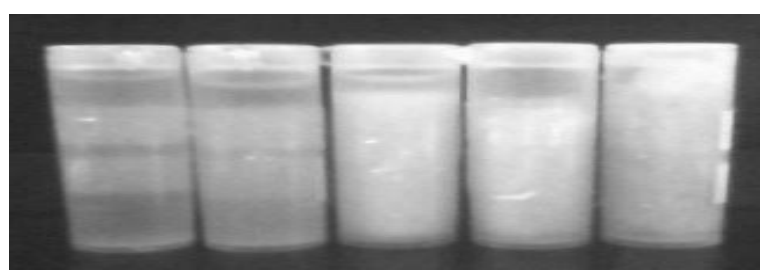

Fig. 3.The physical condition of polymer gels

Based on observation towards the increasing days of the gels being left stand up for almost 23 days, (from left to right, $5^{\text {th }}$ day until $23^{\text {rd }}$ day) (Figure 3 ), the gel are slowly turning from yellow transparent to milky white. Starting from $15^{\text {th }}$ to $20^{\text {th }}$ day and afterwards, there are some white solid appeared inside the gel and the presence increase by the day. This shows that the polymerization was coming to the saturated level. Nevertheless, the values of acoustic speed of the gel were maintained for the last 14 days. Even though the gel went through the polymerization process, the compressibility of the gel does not become greater, as expected. The presence of white solid inside the gel on the $23^{\text {th }}$ days, (Figure $4(\mathrm{~b})$ ), is now considered as 'disturbance' in the ultrasound image.

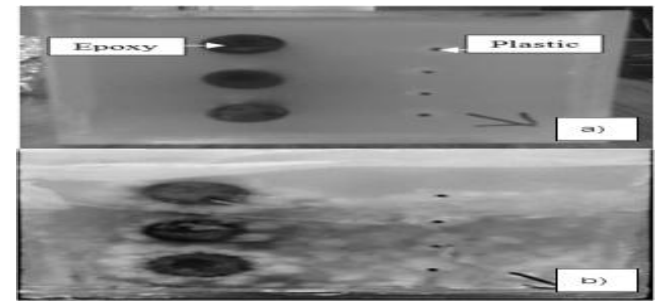

Fig . 4. The substitute materials surrounded by polymer gel inside Perspex container (a) before polymerized (b) after polymerized.

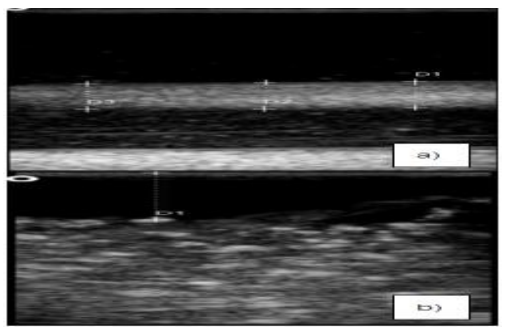

Fig. 5. The ultrasound B-mode image obtained (a) before polymerized (b) after polymerized. The dark background regions show the homogeneous echogenicity of the phantom.
As shown in Figure 5, the ultrasound evaluation was applied by using $10 \mathrm{MHz}$ frequency for the best resolution and contrast to obtain image. Regarding to Figure 5 (a), the image illustrated the epoxy inside the gel in parallel alignment with the linear probe while in Figure 5 (b), the ultrasound image is displayed with lots of small 'bloom' which is originated from Figure 4 (b).

This type of gel is considered match to the acoustic properties of human soft tissue based on the ultrasonic propagation speed and density of the material. Through ultrasound screen, the epoxy material can be seen as the simulation of the human bone.

\section{ACKNOWLEDGEMENT}

The authors acknowledge the supervisor, co-supervisors for their valuable advises and assistance in this research, Dr. Eid Abdel Munem for collimating the alignment of the equipment including explanations regarding to spectrum analysis. The authors would like to appreciate the grant provided by Universiti Sains Malaysia (USM), Penang, Malaysia that has resulted in this article.

\section{REFERENCES}

[1] Anderson PG, Rouze NC, Palmeri ML.Effect of graphite concentration on shear-wave speed in gelatin-based tissue-mimicking phantoms.Ultrason Imaging.33(2):134-42, 2011

[2] Martin O. Culjat, David Goldenberg, Priyamvada Tewari, and Rahul S. Singh A Review of Tissue Substitutes For Ultrasound Imaging Ultrasound in Med. \& Biol., Vol. 36, No. 6, pp. 861-873, 2010

[3] J. E. Brownne, K. V. Ramnarine, A. J. Watson and P.R. Hopkins Assessment of the Acoustic Properties of Common Tissue Mimicking Test Phantom Ultrasound in Med. \& Biol. 2003, 1053-1060

[4] Toshio Kondo and Hiroyuki Fujimoto Ultrasound Tissue-Mimicking Materials using Oil Gel and Measurement of Their Characteristics Jpn. J. Appl. Phys. Vol. 41 (2002) pp. 3598-3599

[5] K Zell, J I Sperl, M W Vogel, R Niessner and C Haisch Acoustical properties of selected tissue phantom materials for ultrasound imaging Phys. Med. Biol. 52 (2007) N475-N484

[6] Kenji Takegami, Yukio Kaneko, Toshiaki Watanabe, Toshiyuki Maruyama, Yoichiro Matsumoto and Hirokazu Nagawa Polyacrylamide Gel Containing Egg White as New Model For Irradiation Experiments Using Focused Ultrasound Ultrasound in Med. \& Biol., Vol. 30, No. 10, pp. 1419-1422, 2004

[7] ElifVargu", $\mathrm{N}$ and Ali Usanmaz Polymerization of 2-Hydroxyethyl Acrylate in Bulk and Solution by Chemical Initiator and by ATRP MethodWileyInterScience2005

[8] Lopez- Haro,S. A., Bazan- Trujillo, I., Leija - Salas, L. and Vera Harnandez, A. Ultrasound propagation speed measurement of mimicking soft tissue phantoms based on Agarose in the range of $25^{\circ} \mathrm{C}$ to $50^{\circ} \mathrm{C} .20085^{\text {th }}$ International Conference on Electrical Engineering, Computing Science and Automatic Control (CCE - 2008). 192-195

[9] J V Trapp, G Michael, Y de Deene and Baldock. Attenuation of diagnostic energy photons by polymer gel dosimeters Phys. Med. Biol. 47 (2002) 4247- 4258

[10] Oudry J, Bastard C, Miette V, Willinger R, Sandrin L. Copolymer-in-oil phantom materials for elastography. Ultrasound Med Biol. 2009

[11] Melissa. L. M et al.Ultrasound evaluation of polymer gel dosimeters. Phys. Med. Biol. 2002, 47 (1449-1458)

[12] V A M Luprano, P A Ramires, G Montagna, and E Milella. Non-destructive characterization of hydrogels. J. Mat. Sci. Mat. Med., 8:175-178, 1997. 